\title{
SECTION INTRODUCTION
}

\section{Session 6 introduction}

John FR Robertson*1 and Per Eystein Lønning*2

Session 6 was entitled 'Who would have thought it!' and comprised four separate presentations that had in common that the inferred conclusions of their titles would not necessarily have been inferred from perceived wisdom.

Professor Smith's presentation was 'Designing adjuvant treatment based on biological measurements in the neoadjuvant setting. He provides informed and personal reflection on the strengths and weaknesses of neoadjuvant therapy both as a research tool and in routine clinical practice. This comes from an expert who is ideally placed to make such observations since the research group at the Royal Marsden Hospital has made a number of significant contributions to this field.

When neoadjuvant therapy was first introduced many researchers and clinicians hoped it would provide better outcomes than standard adjuvant therapy and/or improve breast conservation rates. When longer term outcomes (for example, disease free survival, overall survival) were shown not to be better, attention turned, as Professor Smith highlights, to trying to replace adjuvant with neoadjuvant studies, which would give outcome data many years earlier. In addition, it was thought that neoadjuvant treatment would increase understanding of biological mechanisms of specific agents and help in the development of new therapeutic targets and agents.

Professor Smith gets to the heart of the issue very quickly - the weaknesses associated with response rates (clinical complete response (cCR) or pathological complete response (pCR)) as endpoints. He systematically addresses the problem of pCR with referenced examples: it does not always predict outcome; it only applies to a minority of tumours; it does not apply to oestrogen receptor-positive tumours; it is of no use for endocrine therapy; and you only get the result after the treatment is finished. He then turns his attention to the use of biological markers as predictors of outcome - both short

*Correspondence: john.robertson@nottingham.ac.uk; per.lonning@helse-bergen.no 'Professorial Unit of Surgery, Nottingham City Hospital, Hucknall Road, Nottingham, NG5 1PB, United Kingdom

2Section of Oncology, Institute of Medicine, University of Bergen, and Department of Oncology, Haukeland University Hospital, N-5021, Bergen, Norway term (for example, response) and long term (for example, survival) and shows that these two have not yet been validated. He also makes the important point that most neoadjuvant therapy studies have been performed on larger tumours and that the results may not be transferable to patients with smaller, better prognosis tumours. He does not offer any potential solution to address this latter problem in the neoadjuvant setting.

He finally focuses on short term, peri-operative 'window of opportunity' studies, which potentially provide greater applicability to the majority of patients. While Professor Smith makes a good scientific case for this approach, the potential benefits he highlights remain to be confirmed and validated.

It is now well recognized that in both early and advanced disease the aromatase inhibitors (AIs) are more efficacious than tamoxifen while the side effect profile of AIs is different from tamoxifen. With the latter, a number of the side effects are not preventable and at the same time are potentially life threatening (for example, thrombo-embolic disease, endometrial cancer) while with the AIs the majority are not directly life threatening and are amenable to preventative treatment (musculoskeletal adverse events). The clinical problem is that musculoskeletal side effects are both very common and can be a cause for patients discontinuing AI therapies. Professor Ingle addresses the topic 'Pharmacogenomics explain musculoskeletal adverse events of aromatase inhibitors. His manuscript looks at whether there might be a pharmacogenomic explanation for these musculoskeletal side effects.

It is important to appreciate both the strengths and potential weaknesses of the data presented. For example, the data are based on samples obtained as part of a large, well conducted randomized clinical trial that included two types of AI (a non-steroidal and a steroidal agent) in a matched case control study. On the other hand, the data are based on a single study that is limited mainly to Caucasians. The group did not start with an a priori hypothesis that it sought to prove but rather applied a genome-wide association study as a way of identifying a gene(s) of interest. This approach identified only one SNP of real interest out of over half a million searched. Professor Ingle's group then looked for any known functions associated with this SNP and proposed a link 
between the SNP, the musculoskeletal side effects and chronic inflammation. In the end the researchers come up with a working hypothesis for the mechanism of musculoskeletal side effects associated with AIs. This moves science forward in that we now have a new hypothesis. However, this requires both validation in a different dataset and confirmation that the SNP truly has a causative relationship and not simply one of association.

An issue of debate over the recent years has been the potential clinical importance of genetic variation in tamoxifen metabolism with respect to outcome. It is well known that 4-hydroxytamoxifen and, in particular, 4-hydroxy N-desmethyltamoxifen, first identified by Lien and co-workers in 1989 [1], binds to the oestrogen receptor with an affinity much higher compared to tamoxifen itself. This metabolite, later named endoxifen by Dr Flockhardt and his team [2], has received much attention due to the finding that the key regulatory enzyme in endoxifen production, CYP2D6, harbours a polymorphism separating individuals into poor metabolizers (low endoxifen concentration) versus good metabolizers (high endoxifen concentration).

Conflicting evidence has linked this polymorphism to tamoxifen efficacy in general and to potential drug interactions influencing outcome among tamoxifen users in particular. Multiple compounds, antidepressants in particular, are metabolized by CYP2D6; yet clinical data presented are conflicting. Thus, at the American Society of Clinical Oncology (ASCO) meeting in 2009 two retrospective patient registry studies (one from the US, the other from the Netherlands) addressing the potential impact of CYP2D6-interacting compounds on outcome in patients treated with tamoxifen reached completely opposite conclusions $[3,4]$.

In her presentation, Dr Pritchard carefully reviews pharmacokinetic and pharmacogenetic data related to the issue. Thereafter, she presents a comprehensive survey of clinical evidence with respect to the influence of CYP2D6 polymorphism status on outcome in patients treated with tamoxifen in general and the potential importance of interacting compounds. The influence of selective serotonin reuptake inhibitors (SSRIs) on tamoxifen metabolism may vary between different compounds; evidence suggests a potential detrimental role of paroxetine but not other SSRIs [5]. However, as Dr Pritchard states in her presentation; no consensus has been reached regarding incorporating CYP2D6 genotype testing in routine clinical practise.

The last subject to be discussed during this session was the role of angiogenesis and angiogenesis inhibitors to breast cancer, presented by Dr Harris. While there is a good theoretical rationale for antiangiogenetic therapy [6], so far clinical studies have revealed moderate effects in diseases like ovarian cancer (ASCO 2010) and adjuvant therapy for colorectal cancer (ASCO 2009). Thus, at this years ASCO meeting a combined analysis of the three studies evaluating chemotherapy with and without bevacizumab in metastatic breast cancer revealed a significant improvement with respect to progression-free but not overall survival [7].

In his presentation, Dr Harris defends clinical results achieved with anti-vascular endothelial growth factor (VEGF) therapy, comparing the benefits from VEGF inhibition to other targeted therapies, including ixabepilone as well as AIs. He further provides an elegant overview of the VEGF receptor family, including the potential biological role of mRNA splice variants and, in particular, the potential role of VEGF-2 polymorphisms [8]. He concludes his presentation commenting that antiangiogenic therapy is remarkably active, considering no predictive markers or targets identifying sensitive tumours have been identified. Clearly, the jury is still out, and more data, in particular from translational research studies, are needed before a final verdict may be reached on antiangiogenic therapy in breast cancer.

\section{Abbreviations}

$\mathrm{Al}$, aromatase inhibitor; ASCO, American Society of Clinical Oncology; PCR, pathological complete response; SNP, single-nucleotide polymorphism; SSRI, selective serotonin reuptake inhibitor; VEGF, vascular endothelial growth factor.

\section{Competing interests}

The authors declare that they have no competing interests.

\section{Acknowledgements}

This article has been published as part of Breast Cancer Research Volume 12 Supplement 4, 2010: Controversies in Breast Cancer 2010. The full contents of the supplement are available online at http://breast-cancer-research.com/ supplements/12/S4

Published: 20 December 2010

\section{References}

1. Lien EA, Solheim E, Lea OA, Lundgren S, Kvinnsland S, Ueland PM: Distribution of 4-hydroxy- $\mathrm{N}$-desmethyltamoxifen and other tamoxifen metabolites in human biological fluids during tamoxifen treatment. Cancer Res 1989, 49:2175-2183.

2. Johnson MD, Zuo H, Lee KH, Trebley JP, Rae JM, Weatherman RV, Desta Z, Flockhart DA, Skaar TC: Pharmacological characterization of 4-hydroxy-Ndesmethyl tamoxifen, a novel active metabolite of tamoxifen. Breast Cancer Res Treat 2004, 85:151-159.

3. Aubert RE, Stanek EJ, Yao J, Teagarden JR, Subar M, Epstein RS, Skaar TC, Desta Z, Flockhart DA: Risk of breast cancer recurrence in women initiating tamoxifen with CYP2D6 inhibitors. J Clin Oncol 2009, 27:18s

4. Dezentjé VO, van Blijderveen NJ, Gelderblom H, Putter H, van Herk-Sukel MP, Casparie MK, Egberts AC, Nortier JW, Guchelaar HJ: Effect of concomitant CYP2D6 inhibitor use and tamoxifen adherence on breast cancer recurrence in early-stage breast cancer. J Clin Oncol 2010, 28:2423-2429.

5. Kelly CM, Juurlink DN, Gomes T, Duong-Hua M, Pritchard KI, Austin PC, Paszat LF: Selective serotonin reuptake inhibitors and breast cancer mortality in women receiving tamoxifen: a population based cohort study. BMJ 2010, 340:c693.

6. Folkman J, Hahnfeldt P, Hlatky L: Cancer: looking outside the genome. Nat Rev Mol Cell Biol 2000, 1:76-79.

7. O'Shaughnessy J, Miles D, Gray RJ, Dieras V, Perez EA, Zon R, et al. A metaanalysis of overall survival data from three randomized trials of bevacizumab (BV) and first-line chemotherapy as treatment for patients with metastatic breast cancer (MBC). J Clin Oncol 2010, 28 (Suppl):15s (abstr 1005). 
8. Schneider BP, Wang M, Radovich M, Sledge GW, Badve S, Thor A, Flockhart DA, Hancock B, Davidson N, Gralow J, Dickler M, Perez EA, Cobleigh M, Shenkier T, Edgerton S, Miller KD; ECOG 2100: Association of vascular endothelial growth factor and vascular endothelial growth factor receptor-2 genetic polymorphisms with outcome in a trial of paclitaxel compared with paclitaxel plus bevacizumab in advanced breast cancer: ECOG 2100. J Clin Oncol 2008, 26:4672-4678. doi:10.1186/bcr2744

Cite this article as: Robertson JFR, Lønning PE: Session 6 introduction. Breast Cancer Research 2010, 12(Suppl 4):S15. 